\title{
Los cuatro aspectos esenciales por investigar
}

\author{
The Four Essential Aspects \\ to Investigate
}

\author{
Dr. Ing. José Antonio París (Argentina) \\ Universidad Nacional de La Plata \\ Magíster en Marketing Internacional \\ marketing.jose.paris@gmail.com
}

\begin{abstract}
Resumen
El marketing tradicional angloamericano ofrece una metodología de investigación de mercados simple, aunque no esencial. El costo de asumir esta metodología ha dejado un lamentable saldo de $85 \%$ de probabilidad de fracasos de los planes de marketing lanzados en los mercados de la actualidad. Es de esperarse que esta situación sea inadmisible para las empresas de los mercados latinoamericanos, ya que por su estrecha magnitud de sus mercados y la poca inserción internacional están obligadas a mejorar notablemente su performance investigativa e ir a las cuestiones de fondo, para sentar los cimientos sobre
\end{abstract}

\begin{abstract}
Anglo-American traditional marketing offers a simple, but not essential market research methodology. The cost of assuming this methodology has left a regrettable $85 \%$ balance of failure probability for the marketing plans launched currently. As is to be expected, this situation is unacceptable for companies in the Latin American market because due to their narrow market scale and to the little international incorporation, they are forced to considerably improve their investigative performance and explore in more detail in order to establish the foundation to support their marketing plan. This article presents nine levels of research and promotes
\end{abstract}

$\begin{array}{lrll}\text { Fecha de recepción: } & 4 \text { de marzo de } 2014 & \text { Para citar este artículo / to cite this article } \\ \text { Fecha de revisión: } & 28 \text { de marzo de } 2014 & \text { París, J. A. (2014). Los cuatro aspectos esenciales por } \\ \text { Aprobación: } & 28 \text { de marzo de } 2014 & \text { investigar. Poliantea, 10(18), pp. 65-81. }\end{array}$

París, J. A. (2014). Los cuatro aspectos esenciales por investigar. Poliantea, 10(18), pp. 65-81. 
los que se debe apoyar su plan de marketing. En este artículo, se presentan los nueve niveles de investigación y se promueven técnicas de investigación alternativas que están cambiando los resultados de los planes de marketing que hemos denominado esencial.

Palabras clave: percepción, significado, código, arquetipo, marketing. alternative research techniques that change the results of the marketing plans that are considered essential.

Keywords: perception, meaning, code, archetype, marketing. 


\section{Los cuatro aspectos esenciales por investigar}

\section{The Four Essential Aspects \\ to Investigate}

Dr. Ing. José Antonio París (Argentina)

Universidad Nacional de La Plata

Magíster en Marketing Internacional marketing.jose.paris@gmail.com

\section{Introducción}

A pesar de que la investigación tradicional haya promulgado elocuentes promesas como "leer la mente del consumidor", "interpretar la mente del consumidor" o incluso escuchar "la voz del mercado" (Barobba y Zaltman, 1992), en realidad es muy poco lo que se ha hecho para trabajar adecuadamente en este sentido. Estamos en tiempos en los que se manifiesta la tercera revolución humana, es decir, la revolución de la información. Esta se ha manifestado de manera temprana, pero aún incipiente, por lo cual no se presenta de manera pareja en todas las áreas en las que su impacto es determinante, o incluso con la intensidad que debe y puede alcanzar.

Los efectos de esta revolución son de dos tipos: positivos y negativos. En esencia, lo positivo es que hoy día buena parte del conocimiento humano está al alcance de cualquiera, es decir que prácticamente quien tenga el interés y algunos mínimos recursos podrá conseguir, a partir de la explotación de su curiosidad, que dicha información se convierta en conocimiento. La parte negativa es que, si bien el acceso a la información general está al alcance de todos, si queremos profundizar con dicha información, esta ya no estará al alcance de todos, por lo que la desigualdad social pareciera mantenerse e 
incluso profundizarse. Y en este marco surgen las víctimas de los daños colaterales que genera esta nueva revolución. También las secuelas del posmodernismo, fruto de la revolución industrial, ha dejado un tendal en la sociedad de consumidores creada por el todopoderoso mercado. En este ambiente enrarecido, el hombre ha pasado de ser consumidor a consumido, es decir, el producto por consumir. Al respecto, Zitmunt Bauman (2011) afirma: “... la fuente de las ganancias capitalistas se ha desplazado o ha sido desplazada desde la explotación de la mano de obra fabril hacia la explotación de los consumidores". Lo que dejó como resultado un mundo totalmente deshumanizado en el que la manipulación y la imposición de prácticas de venta hacia los consumidores ha sido una constante.

Por otro lado, desde los sectores más progresistas del capitalismo, se viene anunciando con pompas y platillos desde la década de 1990 que el exceso de información disponible puede llevar a una desorientación generalizada acerca del correcto rumbo por seguir. Tal cual como lo anunciara Toffler (1985): “... cuando hablo de shock del futuro me refiero a la desorientación y sobrecarga de decisiones producidas por el cambio a alta velocidad... [que] a veces lleva a un derrumbamiento de nuestra capacidad de tomar decisiones racionalmente". Quien de alguna manera le dio argumentos a esta afirmación fue Antonio Damasio (2004) en su libro El error de Descartes, quien demostró científicamente que las decisiones empresariales obedecían más a cuestiones emocionales que a cuestiones racionales. Fue desde la neurociencia entonces que se pudo confirmar una ley empírica que ya se conocía del marketing esencial (París, 2009), por la cual se puede afirmar que $95 \%$ de las decisiones de las personas provienen de su inconsciente, y por lo tanto tan solo $5 \%$ de ellas eran realmente racionales. Esto desplazó el mundo de la toma de decisiones hacia el subjetivismo, y el racionalismo hasta ahora "todopoderoso" pasó a un segundo plano, o al menos con aplicaciones muy restrictivas. En este mundo del inconsciente, ahora muy cotizado, tendremos que identificar qué aspectos se deben determinar con certeza.

Para muchos racionalistas, el inconsciente de las personas sigue siendo una "caja negra", difícil de decodificar y casi imposible de estudiar de manera práctica. Por esta razón, y de manera necia, se aferran a las metodologías de investigación 
desarrolladas por el racionalismo del marketing tradicional angloamericano. Los resultados de estas metodologías se visualizan en un lamentable hecho: entre 85 a $90 \%$ de los planes de marketing actuales fracasan, y esto ya había sido denunciado también en la década de 1990 por Kevin J. Clancy y Rovert S. Shulman (1998) en su libro La revolución del marketing, en el que afirman y demuestran de manera estadística que ese fracaso se debe justamente a la aleatoriedad del marketing como disciplina metodológica, ya que $85 \%$ se ajusta a la probabilidad de ocurrencia que Gauss determinara con su curva normal estándar como resultados neutros o malos (lo que en marketing se traduce en pérdidas de tiempo o dinero, que en definitiva como "el tiempo es dinero", entonces, desde el punto de vista mercadológico, es lo mismo).

Desde nuestra óptica del marketing esencial, con una visión más holística y heurística del marketing en la cual hemos integrado los saberes de la filosofía (axiología y semiología), antropología, sociología, psicología, neurociencia, comunicación y comportamiento del consumidor, hemos buscado desmitificar esa "caja negra" que hoy, gracias a los avances de esas ciencias y disciplinas, estamos en condiciones de comprender y analizar con cierto grado de certeza. Y de ello ha surgido que el campo de investigación se debe desarrollar en dos sitios: la mente del consumidor y la mente del mercado. Hacia allí dirigiremos nuestros esfuerzos, ya que comprender ambos campos de acción será el desafío por dilucidar, puesto que lo que descubramos en ellos será la base conceptual de todo plan de marketing esencial.

\section{Los nueve niveles de investigación}

Para comprender la "voz del mercado" primero se requiere conocer e interpretar con claridad las percepciones, significados, códigos y arquetipos de los consumidores que la componen, para luego armonizar creativamente los deseos detectados en el mercado con lo que la compañía es capaz de hacer o producir. Este paso indica la necesidad de contar con la información requerida de acuerdo con el nivel de profundidad que implica el proceso de decisiones del consumidor. Y este a su vez será acorde con los elementos que entren en juego a la hora de decidir, según su complejidad (figura 1). 


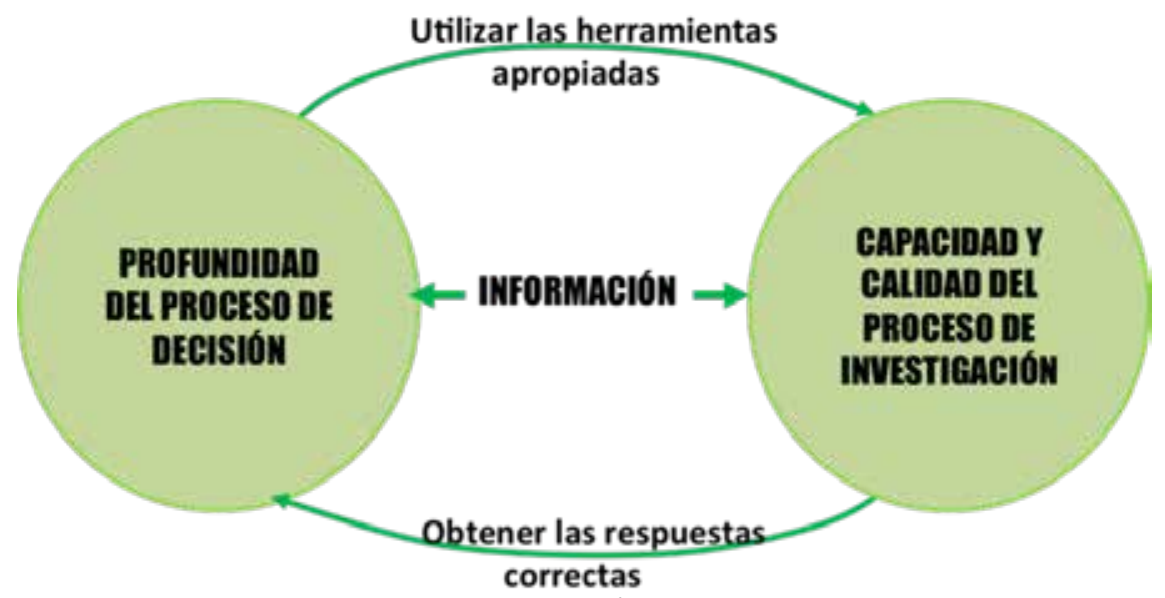

Figura 1. Obtener la información requerida

Fuente: Elaboración propia.

Debido a que de la simulación del bifocal de la figura 1 se desprende que quien toma la decisión es distinto de la quien la suministra, solo se logrará perfeccionar el mercado mediante la aplicación del marketing esencial cuando ambas partes aportan a la visión binocular que a su vez por la combinación de ambos puntos de vista permite una visión estereoscópica en profundidad. Para lo cual, las partes deben aprender a apreciar el punto de vista alternativo. El objetivo último es sin duda lograr un equilibrio armonioso entre ambos puntos de vista. De hecho, cuando los dos puntos de vista, el de la persona como consumidor y de la organización como proveedora, están alineados, en equilibrio y en convergencia, se esperan resultados positivos para ambas partes. Desde el punto de vista del consumidor en el mercado, este podrá tomar mejores decisiones que le permitirán mejorar su calidad de vida y, de alguna manera, promover su felicidad. Y desde el punto de vista de la organización, podrá alcanzar de manera ética y promisoria sus objetivos empresariales y, de esta manera, generar fidedigna y legítima riqueza, sin caer en políticas de imposición o manipulación del mercado.

Ahora, el nivel de profundidad por investigar dependerá de la complejidad del producto o servicio en cuestión y de su relación con el consumidor. Es decir, si el producto se relaciona de manera superficial y solo de manera funcional, la determinación 
de las percepciones será suficiente para identificar el problema en cuestión (es el caso de un taladro eléctrico). Pero, si el producto adquiere ribetes significativos vinculares con terceros, entonces tendremos que determinar su significado en la mente del consumidor (caso de una remera o camiseta con simbolismos estampados o serigrafiados). Avanzando en este sentido, si además se involucran comportamientos sociales (rituales o códigos conductuales, como el comportamiento en un restaurante o en la cola de un banco), entonces deberemos determinar los códigos simbólicos o culturales (dependiendo del caso). Finalmente, si se incorporan a todos los demás cargas energéticas (positivas o negativas) y emociones, es muy posible que tengamos que llegar a figuras del inconsciente colectivo, como los arquetipos (por ejemplo en el consumo del alcohol por parte de los jóvenes tendremos que determinar si el consumo de bebidas alcohólicas convierte al joven en un héroe de la modernidad o en un peligro ambulante).

Sin embargo, nos hemos dispuesto a obtener con nuestras técnicas de investigación una metodología práctica (aunque estas tengan bases subjetivas) que busca hacer aflorar los significados desde el inconsciente de los consumidores (tanto individuales como grupales) en forma de códigos $y$, de esta manera, ofrecer un soporte de bases sólidas al marketing que hemos llamado esencial, ya que se busca llegar a las causas y a la misma esencia del problema. Es decir, trabajar desde sus bases profundas y no desde sus consecuencias como lo viene haciendo el marketing tradicional (figura 2). 


\section{NIVELES DE INVESTIGACIÓN:}

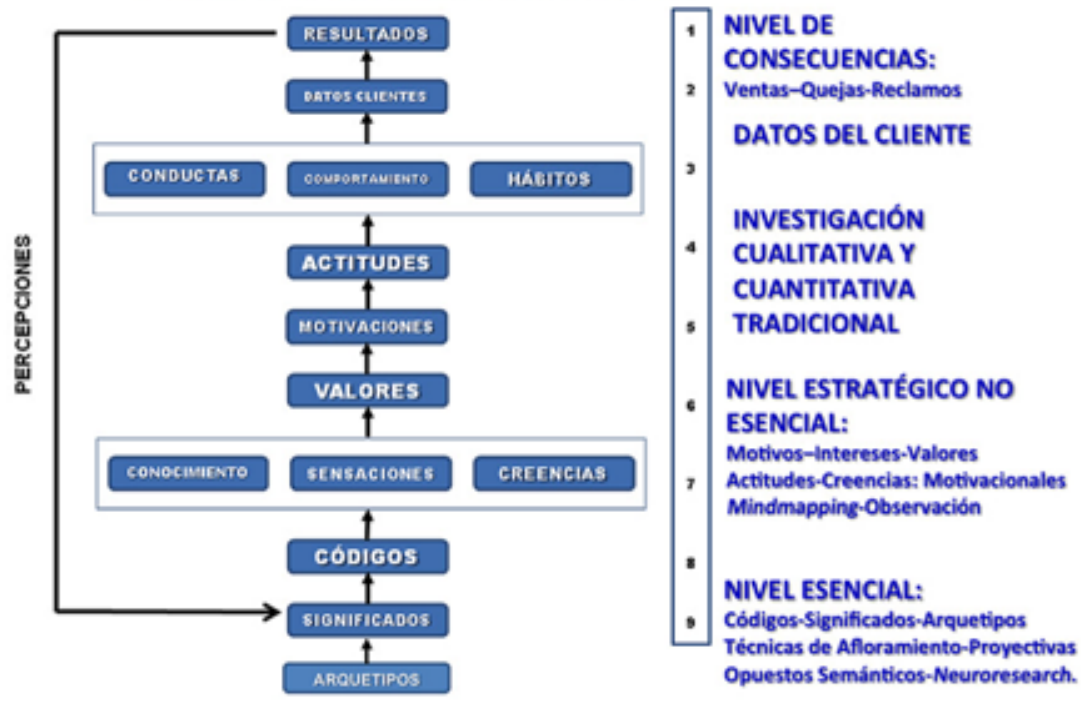

Figura 2. Niveles de investigación.

Fuente: Elaboración propia.

En la figura 2 presentamos nueve niveles de investigación a los que se puede dedicar la metodología de investigación del marketing, los que se describen a continuación:

- Nivel 1 (resultados): estos se miden con un sistema de monitoreo de los indicadores vinculados con las ventas o salidas de las compañías, así como por el análisis de las comentarios, sugerencias, quejas y reclamos de los clientes o usuarios finales de los productos y servicios. Se trata de la respuesta operativa de la compañía a sus acciones de marketing. En esta etapa final, se miden las consecuencias de la implementación del plan de marketing y solo se puede reaccionar a partir de la realización de algunos ajustes de la faz operativa del plan de marketing en cuanto a las acciones de venta (a veces mejorando el sistema de atención o aumentando la agresividad comercial de la compañía). Estas acciones son muy comunes en las empresas que trabajan con el marketing clásico, ya que normalmente refuerzan su actividad comercial de su fuerza de ventas, ya sea 
aumentando su plantilla, ya sea mejorando su capacitación.

Se trata de información racional, de los denominados hechos o "datos ciertos". Una vez analizados estos datos, se convierten en información que proviene del consciente. Casi toda esta información está disponible en la misma organización. Las técnicas para obtener esta información incluyen bases de datos, sistemas de monitoreo, tableros de comando y encuestas de satisfacción a los clientes.

- Nivel 2 (datos de clientes): estos son hechos y características de la cartera de clientes que puede recoger la misma compañía, bien en cada momento de contacto con el cliente, bien por investigación secundaria con la impulsión de referidos y hasta adquiriendo bases de datos externas, para así intensificar sus acciones de ventas con promociones, descuentos o la agresividad comercial de los call centers (ventas telefónicas), entre otras acciones del tipo.

De la misma manera que en el caso anterior, una vez analizados estos datos, se convierten en información consciente y racional. Las técnicas para obtener esta información incluyen sistemas de diagnóstico de situación, tableros de comando y sistemas CRM (customer relationship management).

- Niveles 3 y 4 (actitudes: conductas, comportamiento y hábitos): esta información normalmente proviene de la percepción del cliente y se obtiene a partir de la combinación de métodos cualitativos y cuantitativos. Recordemos que la percepción es el proceso cognoscitivo a través del cual los sujetos captan la información del entorno. $\mathrm{Y}$ es a partir de los sistemas sensoriales que les llega la información y que le permiten confrontarlo con su sistema de referencias internas y así conformar una representación o imagen coherente de su entorno. Se caracteriza por la subjetividad. Ahora, dentro de los métodos cualitativos normalmente se realizan $f o$ cus group ('reuniones de foco') y reuniones de torbellinos de ideas para perfeccionar la información del mercado objetivo en la segunda etapa de investigación (en particular se perfecciona el formulario de preguntas para 
realizar el trabajo de campo). Y luego se trabaja con la investigación cuantitativa, a partir de la realización de encuestas, según una muestra representativa del mercado objetivo. La información obtenida en este nivel resulta de mucha utilidad para realizar ajustes tácticos del plan de marketing, que puede derivar en la modificación de cualquiera de las $4 \mathrm{P}$ del marketing táctico, como modificaciones o adaptaciones en el producto, cambios en la estructura de precios, rediseño de la distribución, relanzamiento de la comunicación y hasta un renovación de la marca fantasía del producto en cuestión. En esta, si se quiere avanzar con información en profundidad, puesto que aquí ya aparecen elementos del consciente, pero muchos más del inconsciente, se debe trabajar con técnicas que permitan determinar con certeza "lo que realmente hace el consumidor" y no solo "lo que dice que hace". Y esto no implica que la gente mienta u omita decir lo que realmente hace o por qué lo hace, es que simplemente no lo sabe de manera racional, ya que estos aspectos son manejados por su inconsciente. Por lo tanto, ya en este nivel deberemos investigar en dos campos: la mente del consumidor y la mente del mercado. Para el primero de dichos campos, proponemos las entrevistas en profundidad. Se tratan de entrevistas muy planificadas, pero que admiten cierta improvisación en el momento de la verdad, es decir, frente al entrevistado. Por lo que se la puede definir como una técnica de “improvisación planificada”, lo importante es conseguir un espacio de diálogo distendido (en realidad se busca un monólogo de parte del entrevistado y el entrevistador solo oficia como orientador de los comentarios del investigado). Con esta técnica se sabe que se está llegando a la profundidad necesaria cuando el entrevistado comenta: "no sabía que sabía lo que te acabo de decir"; con esta señal se sabe que se está llegando al inconsciente de la persona. Y, por lo tanto, la técnica cualitativa está siendo bien utilizada.

Para el segundo de los campos, el de la mente del mercado, desde el marketing esencial utilizamos los estudios motivacionales y la 
observación. Los estudios motivacionales son técnicas cualitativas más profundas que el focus group, aunque ciertamente se requiere un poco más de pericia, en los cuales la acción del coordinador no es tan influyente como en el focus group, pero por encima de todo permite activar las áreas cerebrales con las que los consumidores toman sus decisiones de compra. Además, por medio de esta técnica se pueden determinar relaciones de concomitancia (aspecto importantísimo, ya que se aproxima más a lo que pasa en el mercado), por lo cual esta metodología es mucho más poderosa y eficaz que el focus group. E incluso con el estudio motivacional logramos identificar muchos códigos de conducta del consumidor (algo casi imposible con el focus group).

Por su parte, la observación sea participante o no, grupal o individual, nos permite monitorear lo que realmente hace el consumidor, lo que usa, cómo lo usa, y qué aplicaciones les da a los productos o servicios en cuestión. Aquí se pueden determinar tanto códigos simbólicos como códigos culturales. Recordemos que un código es toda combinación de significados interrelacionados entre sí de manera tal que actúan como filtros del comportamiento de las personas, tipo: "pasa o no pasa". Si son básicos o primitivos, se trata de códigos simbólicos, si por el contrario están diseñados por una determinada cultura de manera convencional, se trata de códigos culturales.

- Niveles 5, 6 y 7: todos estos aspectos provienen del inconsciente de las personas y toman forma de motivaciones, valores, conocimientos, sensaciones y creencias: esta información se obtiene de las metodologías cualitativas propiciadas por el marketing esencial, como estudios motivacionales para las motivaciones (drivers), los estudios etnográficos para los valores y las entrevistas en profundidad y el mindmapping ('mapeo mental') (http://www.tonybuzan.com/) para obtener los conocimientos adquiridos, las sensaciones que producen y las creencias que poseen los consumidores. En este nivel ya se actúa en lo estratégico, ya que las acciones son realizadas 
para identificar cuáles son las motivaciones que llevan al consumidor a tratar de ganar o evitar algo, pues, de lo contrario, el sujeto permanecerá neutro respecto de la conducta que se desea promover. También en este nivel se trata de identificar el nivel de compromiso, es decir: ¿qué es lo que sabe?, ¿qué es lo que piensa?, ¿qué es lo que siente? y ¿qué es lo que cree? Pero, a pesar del nivel de profundidad que se pueda obtener a partir de una buena investigación, nunca se determinará la esencia del problema.

- Niveles 8 y 9 (significados, códigos y arquetipos): a partir de las técnicas de afloramiento de significado (París, 2012) u otras técnicas a las que se pueda acceder para abordar el pensamiento inconsciente del mercado objetivo. Es en este nivel que se llega a la esencia del problema, es decir, se llega a la piedra basal del marketing esencial estratégico. Porque en esta instancia la compañía tiene plena conciencia de lo que realmente pasa en la mente del mercado y del consumidor. Ya que finalmente se comprende ¿qué nos están comprando?, además del ¿para qué? y del ¿por qué? Recordemos que el significado es toda interpretación que una persona de una determinada sociedad y cultura le dará en un determinado momento e, incluso, en una determinada circunstancia a un conjunto de conceptos materiales, inmateriales, o de ambos. Es la representación psíquica de la cosa. Es una creación pura de la mente humana. No son universales, tampoco absolutos, ni permanentes. Ahora, con una carga emotiva muy fuerte, el significado se puede convertir en impronta, y estas tienen una potente presencia en la mente del consumidor y es muy difícil que se resignifique en la mente de la persona que la ha creado. Ahora, los significados y las improntas no pueden dejar de identificarse y determinarse con claridad, porque constituyen la base conceptual de todo plan de marketing esencial.

Ahora, para determinar los arquetipos de Jung (1984), esto lo realizamos cuando los perfiles de los consumidores asumen personajes recurrentes, recurrimos a las técnicas proyectivas y de los opuestos semánticos. Los arquetipos son 
parte del inconsciente profundo colectivo. De allí que las técnicas para determinarlos deben identificar aspectos comunes de una determinada comunidad que definen un determinado personaje. Como el mismo Jung (2005, p. 124) lo afirma: “... no se trata, pues, de representaciones heredadas, sino de posibilidades heredadas de representaciones. Tampoco son herencias individuales, sino, en lo esencial, generales, como se puede comprobar por ser los arquetipos un fenómeno universal". Funcionan como verdaderas cajas vacías (preconformadas y que todos poseemos en su versión positiva o negativa), las que se pueden cargar con energía positiva o negativa dando resultados absolutamente opuestos. Por ejemplo, si observamos cómo funciona el conocimiento, allí podemos observar el grado y la calidad de la información que maneja una persona, diremos que, si esta conoce mucho y en profundidad acerca de determinados temas, tendrá autoridad intelectual para dar opiniones y puntos de vista sobre aquellas temáticas. Por lo cual, según la teoría de los arquetipos, esta persona en dichos temas toma el personaje de sabio; ahora, la misma persona que desconoce la profundidad de muchos otros temas, sin embargo, opina y ofrece teorías sin sentido; aquí esta persona asume el personaje del charlatán (figura 3). Ahora, las figuras arquetípicas solo son requeridas para identificar determinados aspectos del consumo (los que incorporan personajes), pero no siempre es necesaria su determinación. 


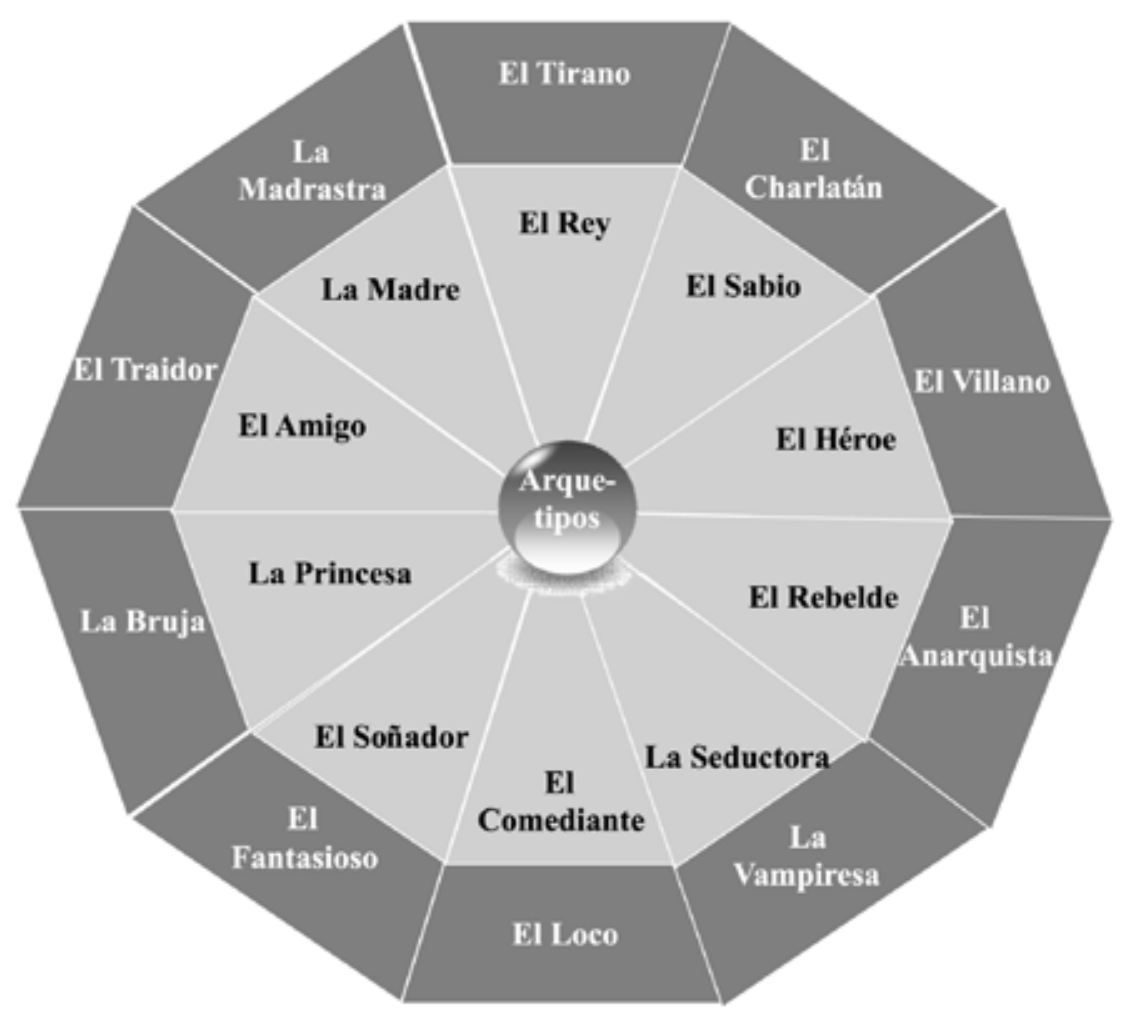

Figura 3. Arquetipos

Fuente: Martínez (2008, pp. 67-80).

\section{Conclusiones}

Un aspecto que debemos aclarar con énfasis es el hecho de que de existir una discrepancia del resultado esperado por el plan de marketing confrontado con la realidad de los resultados mercadológicos logrados se deberá a la fuente del conflicto más natural y corriente del marketing: la percepción de quien ha hecho el plan de marketing no coincide con los significados de base del mercado

objetivo, y eso produce una notable discrepancia que puede llevar al fracaso mercadológico. Para evitar esta situación, hemos creado las técnicas de afloramiento de significados, que buscan darle al investigador una herramienta poderosa para acotar a esa percepción. Aunque debemos aclarar que, si bien seguramente acortaremos la brecha entre el significado en la mente del consumidor con la 
percepción que el investigador logre de ella, igualmente seguirá siendo una percepción, ya que la realidad siempre distará en algo de lo científicamente percibido por el hombre. Según Popper (1985), lo que estamos haciendo con estas técnicas de afloramiento de significados es tirar al "mar de las realidades" una red de trama más fina, para así tratar de pescar una mayor aproximación a la verdad, que en este caso está en la mente del mercado y del consumidor. Pero debemos aclarar que aún seguimos asumiendo que lo que recogerá el investigador especializado seguirá siendo una percepción, que aunque sea más cercana a la realidad y seguramente también más profesional, igualmente seguirá siendo una percepción.

Ahora bien, somos conscientes de que la decisión más racional no implica obtener los mejores resultados, porque en los asuntos humanos casi siempre intervienen el azar y las conjeturas anticipadas que también pueden entorpecer aún más los resultados. Pero, con la experiencia, la pericia y la cantidad de observaciones, la casualidad tiende a equilibrarse y a desaparecer. Pero para ello el investigador debe estar libre de toda parcialidad e interés particular y ser un correcto implementador de las técnicas a los fines de no ser parte del mismo resultado. Por lo que la pericia es un requisito a la hora de aplicar las técnicas de investigación aquí auspiciadas, y por eso la práctica de ellas incrementa notablemente las posibilidades de obtener resultados más precisos, eficaces y también, por qué no, eficientes.

Un aspecto que nos hemos propuesto evitar en esta aplicación de las técnicas de investigación en este trabajo propuestas es el de la irracionalidad de los medios. Ya que desde nuestro punto de vista el objeto científico de este desafío es "ningún fin justifica los medios". Ya hemos dicho que evitaremos la tentadora mezcla de los métodos cualitativos con los cuantitativos, que como nos advierte Schütz (2008), corrompen y confunden al investigador. Por otro lado, en su aplicación se deberá evitar por todos los medios tener una actitud acomodaticia tanto en la etapa de planificación de la tarea investigativa como durante el proceso de investigación, y menos aún en el trabajo de gabinete, en el que se analizarán primero los resultados obtenidos y luego se buscará sintetizarlos.

Luego de estas salvaguardias, finalizaremos este artículo afirmando 
que el nivel de complejidad del proceso de investigación de mercados es mucho más alto que el que se ha promovido desde el enfoque del marketing tradicional angloamericano. Dicha simplicidad no implica calidad, de hecho hoy sabemos que dicho paradigma posee una lamentable tasa de solo $15 \%$ de éxitos. Ahora bien, las técnicas aquí promovidas deberán ajustarse a cada caso y problema particular que se presente. Pero habrá una constante en todas ellas. Todas ellas apuntarán a determinar alguno de los cuatro aspectos relevantes del marketing esencial: percepciones, significados, códigos y arquetipos. Estos deben ser las bases conceptuales de todo plan de marketing esencial.

\section{Referencias bibliográficas}

Barobba, V. y Zaltman, G. (1992). La voz del mercado. Madrid: McGrawHill.

Bauman, Z. (2011). Daños colaterales, desigualdades sociales en la era global. Buenos Aires: Fondo de Cultura Económica.
Clancy, K. J. y Schulman, R. S. (1998).

La revolución del marketing. Buenos Aires: Javier Vergara.

Damasio, A. R. (2004). El error de Descartes, la emoción, la razón y el cerebro humano (3. ${ }^{\mathrm{a}}$ ed.). Barcelona: Crítica.

Jung, C. G. (1984). El hombre y sus símbolos (4. ${ }^{\mathrm{a}} \mathrm{ed}$.). Barcelona: Buc Caralt.

Jung, C. G. (2005). Recuerdos, sueños, pensamientos. Barcelona: Seix Barrall.

Martínez, P. (2008). Cualitativa-mente. Madrid: ESIC.

París, J. A. (2009). Marketing esencial, un enfoque latinoamericano. Buenos Aires: Errepar.

París, J. A. (2012). La segunda miopía del marketing. Buenos Aires: Haber.

Popper, K. E. (1985). La lógica de la investigación científica. Barcelona: Tecnos. 
Schutz, A. (2008). El problema de la realidad social (2. ${ }^{\mathrm{a}}$ ed.). Buenos Aires: Amorrortu.

Tony Buzan: mindmapping. Recuperado de http://www.tonybuzan.com/ Toffler, A. (1985). Entrevistado por J. W. Thompson. Recuperado de http:// www.jwt.com/ 